\title{
Two meshless methods for solving nonlinear ordinary differential equations in engineering and applied sciences
}

https://doi.org/10.1515/nleng-2020-0012

Received Jul 22, 2019; accepted Mar 11, 2020.

\begin{abstract}
In this paper, two meshless methods have been introduced to solve some nonlinear problems arising in engineering and applied sciences. These two methods include the operational matrix Bernstein polynomials and the operational matrix with Chebyshev polynomials. They provide an approximate solution by converting the nonlinear differential equation into a system of nonlinear algebraic equations, which is solved by using Mathematica ${ }^{\circledR}$ 10. Four applications, which are the well-known nonlinear problems: the magnetohydrodynamic squeezing fluid, the Jeffery-Hamel flow, the straight fin problem and the Falkner-Skan equation are presented and solved using the proposed methods. To illustrate the accuracy and efficiency of the proposed methods, the maximum error remainder is calculated. The results shown that the proposed methods are accurate, reliable, time saving and effective. In addition, the approximate solutions are compared with the fourth order Runge-Kutta method (RK4) achieving good agreements.
\end{abstract}

Keywords: nonlinear differential equations; approximate solution; Bernstein polynomials; Chebyshev polynomials; magnetohydrodynamic squeezing fluid; Jeffery-Hamel flow

\section{Introduction}

Nonlinear ordinary differential equations (NODE) play a significant role in all branches of science and engineering. Many material events can be formulated by the differential

\footnotetext{
*Corresponding Author: Majeed A. Al-Jawary, Department of Mathematics, College of Education for Pure Sciences (Ibn AL-Haitham) / University of Baghdad, Baghdad, Iraq,

E-mail: majeed.a.w@ihcoedu.uobaghdad.edu.iq

Ghada H. Ibraheem, Department of Mathematics, College of Education for Pure Sciences (Ibn AL-Haitham) / University of Baghdad, Baghdad, Iraq, E-mail: ghada.h@ihcoedu.uobaghdad.edu.iq
}

equation. These types of equations appear in a wide range of problems including, but not limited to, fluid mechanics, chemical matters, electricity and electronics. Therefore, the need for reliable and effective method to solve this kind of equation has become a very important requirement [1].

Orthogonal functions and polynomials are instruments extremely helpful in approximation theory and numerical analysis [2].The main feature of this technique is to simplify the solution by converting the equation into a system of algebraic equations. Accordingly, simplifying these problems substantially, and approximate the unknown function by using the polynomial series and then using the operational matrices to get rid of the integration and differentiation.

In the last few years, there was much research done by using the Bernstein polynomials. Bernstein polynomials were introduced in [2], which are used to solve the differential equations. In Ref. [3] authors proposed the collocation method by Bernstein polynomials to solve the variational problems. Also, Alshbool et al. presented the approximate solution of singular nonlinear differential equations by using a collocation method and Bernstein polynomials [4]. Moreover, the Bernstein polynomials have been successfully employed for a class of boundary value problems [5]. In addition, Khataybeh et al. havesolved directly third- order ODEs by using the Bernstein operational matrices method [6].

On the other hand, there are several works dealing with the use of the Chebyshev operational matrix method. Sharma et al. have implemented the Chebyshev operational method to solve Lane-Emden problem [7]. Also, Öztür was studied the solution of the system of differential equations by using the Chebyshev operational matrix method. Rajeev and Raigar have used a numerical approach based on the Chebyshev wavelets operational matrix to solve Stefan problem [9]. Recently, Hashemizadeh, and Mahmoudi have used shifted Chebyshev operational matrix to solve the Physiology problems [10]. 
The main objective of this paper is to implement two meshless methods the operational matrices methods based on Bernstein polynomials and the Chebyshev polynomials to solve some NODE that appear in engineering and applied sciences.

This paper has been organized as follows: In Section 2 the Bernstein polynomials and their operational matrices are introduced. In Section 3 the first kind shifted Chebyshev polynomials and their operational matrices are considered. In Section 4, some nonlinear problems will be introduced and solved by using the proposed methods. Finally, the conclusion will be given in Section 5.

\section{Bernstein polynomials}

The $n$th degree of Bernstein Polynomials on the interval [0, 1] are defined by [11]:

$$
B_{i, n}(x)=\left(\begin{array}{c}
n \\
i
\end{array}\right) x^{i}(1-x)^{n-i}, 0 \leq i \leq n,
$$

where

$$
\left(\begin{array}{c}
n \\
i
\end{array}\right)=\frac{n !}{i !(n-i) !} \text {. }
$$

There are $n+1$, nth degree Bernstein Polynomials. For mathematical convenience, the equation $B_{i, n}(x)=0$ if $i<$ 0 or $n<i$ is often used. These polynomials have many useful and important properties making it beneficial, some of these properties in the following list [11]

(a) Positivity property:

$B_{i, n}(x)>0$ for all $i=0,1, \ldots, n$ and all $x \in[0,1]$.

(b) Unity partition property

$$
\sum_{i=0}^{n} B_{i, n}(x)=\sum_{i=0}^{n-1} B_{i, n-1}(x)=\ldots=\sum_{i=0}^{1} B_{i, 1}(x)=1 .
$$

(c) Recursion's relation property:

$$
B_{i, n}(x)=(1-x) B_{i, n-1}(x)+x B_{i-1, n-1}(x) .
$$

It is easily to approximate any polynomial of degree $n$ to the form of linear combination as given below,

$$
y(x)=\sum_{i=0}^{n} C_{i} B_{i, n}(x)=C^{T} \Phi(x),
$$

where $C^{T}=\left[C_{0}, C_{1}, \ldots, C_{n}\right]$ and $\Phi(x)=\left[B_{0, n}, B_{1, n}, \ldots, B_{n, n}\right]^{T}$

Also, we could make the disintegration of the vector $\Phi(x)$ as a multiplication of a square matrix of size $(n+1) \times(n+1)$ and vector $(n+1) \times 1$ i.e. $\Phi(x)=A X$, where

$$
X=\left[\begin{array}{c}
1 \\
x \\
x^{2} \\
\vdots \\
x^{n}
\end{array}\right]
$$

Define vector $A_{i+1}$

$$
A_{i+1}=[\overbrace{0,0, \ldots, 0}^{i \text {-times }},(-1)^{0}\left(\begin{array}{l}
n \\
i
\end{array}\right),(-1)^{1}\left(\begin{array}{l}
n \\
i
\end{array}\right)\left(\begin{array}{l}
n-i \\
1
\end{array}\right), \ldots,(-1)^{n-i}\left(\begin{array}{l}
n \\
i
\end{array}\right)\left(\begin{array}{c}
n-i \\
n-i
\end{array}\right)]
$$


for $i=0,1, \ldots, n$.

Now if we define $(n+1) \times(n+1)$ matrix $A$ such that $A=\left[\begin{array}{c}A_{1} \\ A_{2} \\ \vdots \\ A_{n+1}\end{array}\right]$, we obtain
$A=\left[\begin{array}{cccc}(-1)^{0}\left(\begin{array}{l}n \\ 0\end{array}\right) & (-1)^{1}\left(\begin{array}{c}n \\ 0\end{array}\right)\left(\begin{array}{l}n-0 \\ 1\end{array}\right) & \ldots & (-1)^{n-0}\left(\begin{array}{c}n \\ 0 \\ 0\end{array}\right. \\ (-1)^{0}\left(\begin{array}{l}n \\ i\end{array}\right) & \ldots & (-1)^{n-i}\left(\begin{array}{c}n-0 \\ n \\ i\end{array}\right)\left(\begin{array}{c}n-0 \\ n-i \\ n-i\end{array}\right) \\ \vdots & \ddots & \\ 0 & 0 & \cdots & (-1)^{0}\left(\begin{array}{c}n \\ n\end{array}\right)\end{array}\right]_{(n+1) \times(n+1)}$

The matrix $A$ is an upper triangular matrix and $|A|=\prod_{i=0}^{n}\left(\begin{array}{c}n \\ i\end{array}\right)$, so that $A$ is an invertible matrix.

\subsection{Operational matrix of product for Bernstein polynomials}

In Section 2, we obtain a clear formula for the Bernstein polynomials of $n$-th degree, now let us introduce the operational matrix of product.

Let $D$ is an $(n+1) \times(n+1)$ operational matrix of the derivative, then

$$
\frac{d}{d x} \phi(x)=D \phi(x), \quad 0 \leq x \leq 1
$$

The matrix $D$ is given by $D=A \sigma A^{-1}$, where $\sigma$ is $(n+1) \times(n)$ matrix [2]

$$
\sigma=\left[\begin{array}{ccccc}
0 & 0 & 0 & \ldots & 0 \\
1 & 0 & 0 & \ldots & 0 \\
0 & 2 & 0 & \ldots & 0 \\
\vdots & \vdots & \vdots & \ddots & 0 \\
0 & 0 & 0 & \ldots & n
\end{array}\right]
$$

Equation (5) can be written for higher derivative as follows

$$
\frac{d^{n}}{d x^{n}} \phi(x)=(D)^{n} \phi(x) \text { where } n=1,2, \ldots
$$

Now, we can use this operational matrix to solve the equation by approximate the unknown function $y(x)$ as $y(x)=$ $C^{T} \Phi(x)$ and we have

$$
y^{\prime}(x)=C^{T} D \Phi(x), y^{\prime \prime}(x)=C^{T}(D)^{2} \Phi(x), \ldots, y^{(n)}(x)=C^{T}(D)^{n} \Phi(x) .
$$

This approximation is applied to all conditions of the equation as well.

We select the Chebyshev roots as the collocation node,

$$
x_{i}=\frac{1}{2}\left(\cos \left[\frac{i \cdot \pi}{n}\right]+1\right), \quad i=0,1, \ldots, n-j \text { where } j=n-1
$$

We will change the unknown functions and their derivatives using Eqs. (2) and (8), and then replace the nodes in its. The resulting were compensated in the NODE to obtain a system of algebraic equations that we so resolve by computer programs such as Mathematica or MATLAB. The convergent analysis of this method was illustrated by [2]. 


\section{First kind Chebyshev polynomials and the operational matrices}

The shifted Chebyshev polynomials (the first kind) $P_{n}(x)$ of degree $n$ is defined by [12]

$$
P_{n}(x)=\sum_{k=0}^{n}(-1)^{n-k} 2^{2 k} \frac{(n+k-1) !}{(n-k) !(2 k) !}(x)^{k}
$$

The unknown function $y(x) \in L^{2}[0,1]$ can be approximated as the form

$$
y(x)=\sum_{i=0}^{\infty} c_{i} P_{i}(x)
$$

where $c_{i}=\left\langle y(x), P_{i}(x)\right\rangle=(2 i+1) \int_{0}^{1} y(x) P_{i}(x) d x \quad i=$ $0,1, \ldots$

In general, we consider only the first $(n+1)$-terms of the shifted Chebyshev polynomials. Hence, we write

$$
y(x)=\sum_{i=0}^{n} c_{i} P_{i}(x)=C^{T} \Phi(x)
$$

where $C^{T}=\left[c_{0}, c_{1}, \ldots, c_{n}\right]$ and $\Phi(x)=$ $\left[P_{0}(x), P_{1}(x), \ldots, P_{n}(x)\right]^{T}$

Currently, we consider the derivative of the vector $\Phi(x)$ by

$$
\frac{d}{d x} \phi(x)=D^{\star} \phi(x)
$$

where $D^{\star}$ is $(n+1) \times(n+1)$ operational matrix of derivative given by

$$
\begin{aligned}
& D^{\star}= \\
& \left\{\begin{array}{c}
\frac{4 i}{\eta_{j}} \\
0
\end{array} \quad i-k\left\{\begin{array}{cl}
k=1,3,5, \ldots, n-1 & \text { if } n \text { is even } \\
k=1,3,5, \ldots, n & \text { if } n \text { is odd } \\
\text { otherwise }
\end{array}\right.\right.
\end{aligned}
$$

where $\eta_{0}=2$ and $\eta_{k}=1$ for $k \geq 1$ [7].

If $n$ is even the matrix $D^{\star}$ defined as fallows

$$
D^{\star}=\left[\begin{array}{ccccccc}
0 & 0 & 0 & 0 & \ldots & 0 & 0 \\
2 & 0 & 0 & 0 & \ldots & 0 & 0 \\
0 & 8 & 0 & 0 & \ldots & 0 & 0 \\
6 & 0 & 12 & 0 & \ldots & 0 & 0 \\
0 & 16 & 0 & 16 & \ldots & 0 & 0 \\
\vdots & \vdots & \vdots & \vdots & \ddots & 0 & 0 \\
2 n-2 & 0 & 4 n-4 & 0 & \ldots & 0 & 0 \\
0 & 4 n & 0 & 4 n & \ldots & 4 n & 0
\end{array}\right]
$$

Also, if $n$ is odd then $k=1,3,5, \ldots, n$ and the matrix $D^{\star}$ can be written as

$$
D^{\star}=\left[\begin{array}{ccccccc}
0 & 0 & 0 & 0 & \ldots & 0 & 0 \\
2 & 0 & 0 & 0 & \ldots & 0 & 0 \\
0 & 8 & 0 & 0 & \ldots & 0 & 0 \\
6 & 0 & 12 & 0 & \ldots & 0 & 0 \\
0 & 16 & 0 & 16 & \ldots & 0 & 0 \\
\vdots & \vdots & \vdots & \vdots & \ddots & 0 & 0 \\
0 & 4 n-4 & 0 & 4 n-4 & \ldots & 0 & 0 \\
2 n & 0 & 4 n & 0 & \ldots & 4 n & 0
\end{array}\right]
$$

By using Eq. (13), we can write the $n$-th derivative as

$$
\frac{d^{n}}{d x^{n}} \phi(x)=\left(D^{\star}\right)^{n} \phi(x) \text {. }
$$

Thus, the operational matrix can be applied to solve the NODE by approximate the unknown function $y(x)=$ $C^{T} \Phi(x)$ and their derivatives by

$$
\begin{aligned}
& y^{\prime}(x)=C^{T} D^{\star} \Phi(x), y^{\prime \prime}(x)=C^{T}\left(D^{\star}\right)^{2} \Phi(x), \ldots, \\
& y^{(n)}(x)=C^{T}\left(D^{\star}\right)^{n} \Phi(x) .
\end{aligned}
$$

We will replace unknown functions and derivatives using Eqs. (12) and (17), and then they are compensated in NODE and their conditions, we then assign the contract in these equations to obtain a system of nonlinear equations $(n+1)$ which can be solved using by the computer programs Mathematica or MATLAB to get the coefficients of vector $C^{T}$.

\section{Test problems}

In this section, the Bernstein and Chebyshev operational matrices methods will be implemented to solve some problems of NODEs that appear in engineering and applied sciences.

\subsection{Magnetohydrodynamic (MHD) squeezing fluid flow}

(15a) The squeezing fluid flow in a porous medium with magnetic field influence shows in Figure 1 has the governing equations [13]:

$$
\begin{gathered}
\nabla \cdot \mathbf{W}=0, \\
\rho D \mathbf{W}=\nabla \cdot \mathbf{T}+(\mathbf{J} \times \mathbf{B})+\mathbf{r} .
\end{gathered}
$$


where $\mathbf{W}$ is the velocity vector, $\mathbf{J}$ the electric current density and $\mathbf{B}=\mathbf{B}_{0}+\mathbf{b}$ is the total magnetic field and $\mathbf{B}_{0}, \mathbf{b}$ are the imposed and induced magnetic fields, respectively.

$\mathbf{T}=-p \mathbf{I}+\mu \mathbf{A}$ is the Cauchy stress tensor with $\mathbf{A}=\nabla \mathbf{W}+$ $(\nabla \mathbf{W})^{t}$ and $\mathbf{r}$ is the Darcy's resistance.

The MHD force $\mathbf{J} \times \mathbf{B}$ can be written as follows:

$$
\mathbf{J} \times \mathbf{B}=-\sigma \mathbf{B}_{0}^{2} \mathbf{W} .
$$

Suppose that the magnetic field is enforced along $\mathrm{z}$ axis and the plates are non conducting. For small velocity $w$, the gap distance $2 L$ between the panels slowly changes over time $t$ so that it can be used as a constant.

For axial symmetry, the components $W$ for the present case are $W=\left(w_{r}, 0, w_{z}\right)$. If the generalized pressure given by $P=\rho\left(w_{r}^{2}+w_{z}^{2}\right)+p$ and the flow are steady then the Navier-Stokes Eq. (18) can be written as

$$
\begin{gathered}
\frac{\partial P}{\partial r}-\rho\left(\frac{\partial w_{z}}{\partial r}-\frac{\partial w_{r}}{\partial z}\right) w_{z} \\
=-\left[\mu \frac{\partial}{\partial z}\left(\frac{\partial w_{z}}{\partial r}-\frac{\partial w_{r}}{\partial z}\right)+\left(\frac{\mu}{k}+\sigma B_{0}^{2}\right) w_{r}\right], \\
\frac{\partial P}{\partial z}-\rho\left(\frac{\partial w_{z}}{\partial r}-\frac{\partial w_{r}}{\partial z}\right) w_{r} \\
=-\frac{\mu}{r} \frac{\partial}{\partial r}\left[r\left(\frac{\partial w_{z}}{\partial r}-\frac{\partial w_{r}}{\partial z}\right)\right]-\frac{\mu}{\kappa} w_{z} .
\end{gathered}
$$

Using the transformation $\psi(r, z)=r^{2} f(z)$ [14], eliminating the pressure $P$ from Eqs. (19) and (20), we get

$$
f^{i v}(z)-\left(\frac{1}{k}+\frac{\sigma B_{0}^{2}}{\mu}\right) f^{\prime \prime}(z)+2 \frac{\rho}{\mu} f^{\prime \prime \prime}(z)=0,
$$

with boundary conditions

$$
f(0)=0, f^{\prime \prime}(0)=0, \quad f(L)=\frac{v}{2}, \quad f^{\prime}(L)=0 .
$$

Using the following dimensionless parameters

$$
F^{\star}=\frac{2 f}{v}, \quad z^{\star}=\frac{z}{L}, \quad R=\frac{\rho h v}{\mu}, \quad m=L \sqrt{\frac{1}{k}+\frac{\sigma B_{0}^{2}}{\mu}} .
$$

and omitting the $\left({ }^{\star}\right)$ then the Eqs. (21) and (22) become

$$
\begin{gathered}
F^{i v}(z)-m^{2} F^{\prime \prime}(z)+R F(z) F^{\prime \prime \prime}(z)=0, \\
F(0)=0, \quad F^{\prime \prime}(0)=0, \quad F(1)=1, \quad F^{\prime}(1)=0 .
\end{gathered}
$$

In our work, we take $m=R=1$.

\subsubsection{Solving the MHD squeezing fluid by Bernstein and Chebyshev operational matrices methods}

The two meshless methods will be applied to solve the first problem by using the Bernstein and first kind shifted Chebyshev operational matrices.

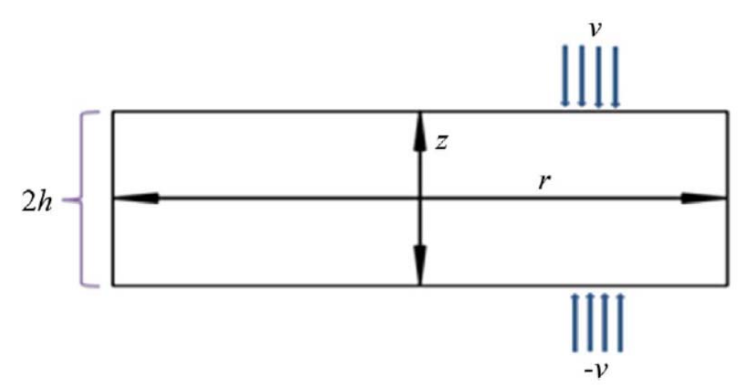

Figure 1: Geometry of the flow [13]

The first step, let us write the function $F(z)$ and its derivatives as matrices by using Eqs. (2) and (8), for the Bernstein polynomial, we get

$$
\begin{gathered}
C^{T}(D)^{4} \Phi(z)-m^{2} C^{T}(D)^{2} \Phi(z)+R C^{T} \Phi(z) C^{T}(D)^{3} \Phi(z)=0 \\
C^{T} \Phi(0)=0, \quad C^{T}(D)^{2} \Phi(0)=0 \\
C^{T} \Phi(1)=1, \quad C^{T} D \Phi(1)=0 .
\end{gathered}
$$

Also, by using the Eqs. (12) and (17) for the Chebyshev polynomial, we obtain

$$
\begin{aligned}
& C^{T}\left(D^{\star}\right)^{4} \Phi(z)-m^{2} C^{T}\left(D^{\star}\right)^{2} \Phi(z) \\
& +R C^{T} \Phi(z) C^{T}\left(D^{\star}\right)^{3} \Phi(z)=0, \\
& C^{T} \Phi(0)=0, \quad C^{T}\left(D^{\star}\right)^{2} \Phi(0)=0, \\
& C^{T} \Phi(1)=1, \quad C^{T} D^{\star} \Phi(1)=0 .
\end{aligned}
$$

To find the values of the unknown coefficients $C^{T}=$ $\left[c_{0}, c_{1}, \ldots, c_{n}\right]$ we get the algebraic system by substitute the collocation nodes in Eq. (9) into Eq. (26) or Eq. (27) and solve it.

The following approximate polynomial for this problem when $n=12, R=1, m=1$, will be obtained:

- By using Bernstein polynomial operational matrices

$$
\begin{aligned}
& F(z) \approx 0+1.50812717 z-0.51862906 z^{3} \\
& -0.000017601 z^{4}+0.013252656 z^{5}-0.000225471 z^{6} \\
& -0.002559665 z^{7}-0.0006791969 z^{8}+0.001103192 z^{9} \\
& -0.0004403875 z^{10}+0.000074039 z^{11} \\
& -0.000005673 z^{12}
\end{aligned}
$$

- By using shifted Chebyshev polynomial operational matrices 
$F(z) \approx 1.973460121 \times 10^{-11}+1.50812716 z$

$+1.93756 \times 10^{-7} z^{2}-0.51863211 z^{3}$

$+0.0000071323 z^{4}+0.01313555 z^{5}$

$+0.0001217442 z^{6}-0.003225565 z^{7}$

$+0.00015101 z^{8}+0.000445903 z^{9}-0.0001310256 z^{10}$

In order to inspect the accuracy for the solution obtained by suggested methods for Eq. (24), we calculate the maximal error remainder $M E R_{n}$ since the exact solution is unknown.

The error remainder function for MHD squeezing fluid can be defined as follows

$$
E R_{n}(z)=F^{i v}(z)-F^{\prime \prime}(z)+F(z) F^{\prime \prime \prime}(z),
$$

and the $M E R_{n}$ is

$$
M E R_{n}=\max _{0 \leq z \leq 1}\left|E R_{n}(z)\right|
$$

Figure 2 shows that the logarithmic plots for $M E R_{n}$ of the approximate solution obtained by the methods of Bernstein and Chebyshev operational matrices which indicate the efficiency of these methods. In Figure 2 we taken $n=6,7,8,9,10,11$, we can see the efficiency by increasing $n$, the errors will be decreasing, this can be described in both cases.

Moreover, Figure 2 presents the method of the operational matrix which depends on Bernstein polynomial is more effective than the Chebyshev polynomial.

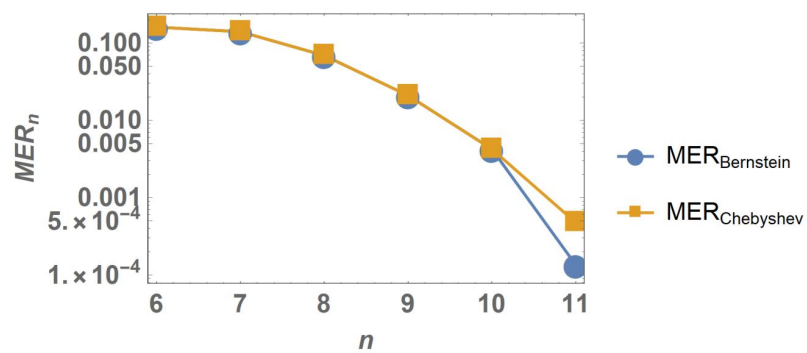

Figure 2: Logarithmic plots for the $M E R_{n}$ for versus $\mathrm{n}$ for MHD squeezing fluid problem

Further numerical investigation can be made, a numerical comparison has been made between the solutions obtained the suggested methods and the Range- Kutta (RK4) method when $n=12$. This comparison is illustrated in Figure 3, it can be seen a good agreements between the approximate solutions and RK4.

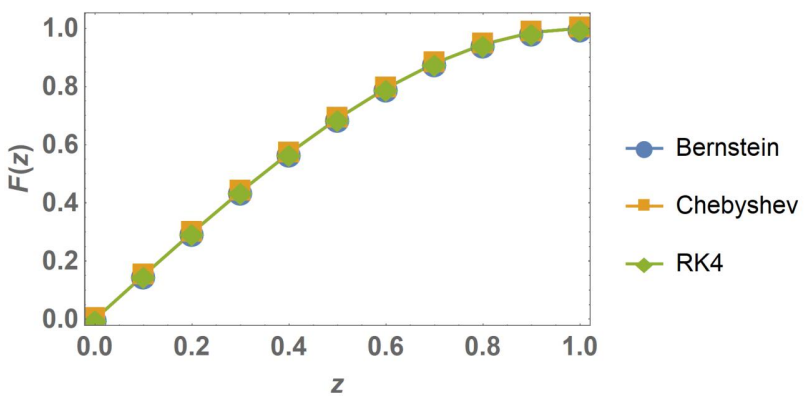

Figure 3: The comparison of the solutions for MHD squeezing fluid problem when $n=12, R=1, m=1$.

\subsection{Jeffery- Hamel fluid flow}

The flow between two nonparallel walls is one of the most important problems in fluid mechanics because of the wide range of applications [15].

The governing equations for the Jeffery-Hamel flow incompressible viscous fluid that is present at the intersection of two rigid, nonparallel plane walls; angle between walls is $2 \alpha$. Flow is supposed to be purely radial and symmetric. Thus the velocity field can be denoted by $V=\left[u_{r}, 0,0\right]$ where $u_{r}=u(r, \theta)$ is the velocity along radial direction, see Figure 4 [16].

The continuity and Navier-Stokes equations in the polar coordinates can be written as follows

$$
\frac{\rho}{r} \frac{\partial}{\partial r}(r u(r, \theta))=0
$$

$$
\begin{aligned}
& u(r, \theta) \frac{\partial u(r, \theta)}{\partial r}=-\frac{1}{\rho} \frac{\partial p}{\partial r} \\
& +v\left[\frac{\partial^{2} u(r, \theta)}{\partial r^{2}}+\frac{1}{r} \frac{\partial u(r, \theta)}{\partial r}+\frac{1}{r^{2}} \frac{\partial^{2} u(r, \theta)}{\partial \theta^{2}}-\frac{u(r, \theta)}{r^{2}}\right] \\
& -\frac{\sigma B_{0}^{2}}{\rho r^{2}} u(r, \theta), \\
& -\frac{1}{\rho r} \frac{\partial p}{\partial r}+\frac{2 v}{r^{2}} \frac{\partial u(r, \theta)}{\partial \theta}=0
\end{aligned}
$$

where $B_{0}$ represents the electromagnetic induction and $\sigma, p, \rho, v$ are the conductivity of the fluid, pressure gradient, constant density of fluid and kinematic viscosity respectively.

We can written Eq.(30) as the form

$$
f(\theta)=r u(r, \theta)
$$

Dimensionless parameters are defined by [15]

$$
F(\eta)=\frac{f(\theta)}{f_{\max }}, \text { where } \eta=\frac{\theta}{\alpha} .
$$


By eliminating pressure terms from Eqs. (31) and (32) and using the formulations in Eqs. (33) and (34), we obtain a third- order differential equation:

$$
F^{\prime \prime \prime}(\eta)+2 \alpha \operatorname{Re} F(\eta) F^{\prime}(\eta)+(4-H a) \alpha^{2} F^{\prime}(\eta)=0,
$$

with the boundary conditions as bellow

$$
F(0)=1, F^{\prime}(0)=0, \quad F(1)=0 .
$$

where the Reynolds number $R e=\frac{\alpha U_{\max }}{v}$, and $H a^{2}=\frac{\sigma B_{0}^{2}}{\rho v}$ is the square of the Hartmann number.

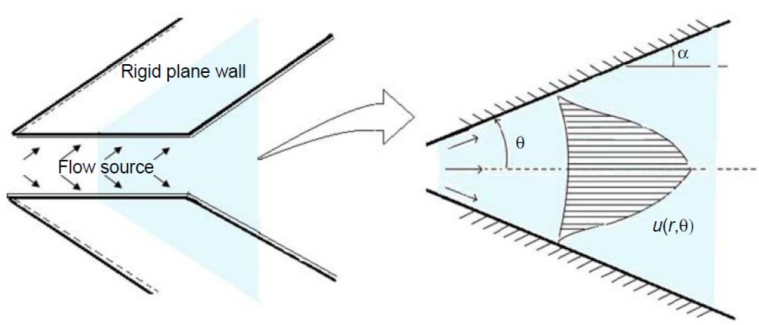

Figure 4: The geometry of the problem [16]

\subsubsection{Solving Jeffery-Hamel fluid flow by Bernstein and Chebyshev operational matrix methods}

The procedures for the methods of the operational matrices based on Bernstein and Chebyshev polynomials can be applied to solve the Eq. (35) with boundary conditions Eq. (36).

Replace the $F(\eta)$ and its derivatives as matrices by using Eqs. (2) and (8), for the Bernstein polynomial, we get

$$
\begin{aligned}
& C^{T}(D)^{3} \Phi(\eta)+2 \alpha \operatorname{Re} C^{T} \Phi(\eta) C^{T} D \Phi(\eta) \\
& \quad+(4-H a) \alpha^{2} C^{T} D \Phi(\eta)=0, \\
& C^{T} \Phi(0)=1, \quad C^{T} D \Phi(0)=0, \quad C^{T} \Phi(1)=0 .
\end{aligned}
$$

If we use the Eqs. (12) and (17) for the Chebyshev polynomial, we obtain

$$
\begin{aligned}
& C^{T}\left(D^{\star}\right)^{3} \Phi(\eta)+2 \alpha \operatorname{Re} C^{T} \Phi(\eta) C^{T} D^{\star} \Phi(\eta) \\
& +(4-H a) \alpha^{2} C^{T} D^{\star} \Phi(\eta)=0,
\end{aligned}
$$

$$
C^{T} \Phi(0)=1, \quad C^{T} D^{\star} \Phi(0)=0, \quad C^{T} \Phi(1)=0 .
$$

Calculate the values of $C^{T}=\left[c_{0}, c_{1}, \ldots, c_{n}\right]$ by solving the algebraic system obtained by substitute the collocation nodes on the Eqs. (37) and (38).
The following approximate polynomial for this problem when $n=12, \alpha=5^{\circ}, R e=10, H a=0$ will be obtained

- By using Bernstein polynomial operational matrices

$$
\begin{aligned}
& F(\eta) \approx 1 .-1.125974388 \eta^{2}+0.000001614788 \eta^{3} \\
& +0.1666094857 \eta^{4}+0.00008832969 \eta^{5} \\
& -0.047086414 \eta^{6}+0.000926031 \eta^{7} \\
& +0.00557524719 \eta^{8}+0.00234250455 \eta^{9} \\
& -0.0035943983 \eta^{10}+0.00129205 \eta^{11} \\
& -0.000180064 \eta^{12}
\end{aligned}
$$

- By using shifted Chebyshev polynomial operational matrices

$$
\begin{aligned}
& F(\eta) \approx 1.0000000000214-6.174114950 \times 10^{-9} \eta \\
& -1.125974093223 \eta^{2}-0.0000038895835 \eta^{3} \\
& +0.1666625548 \eta^{4}-0.0002135427176 \eta^{5} \\
& -0.0459979297696 \eta^{6}-0.001657455901 \eta^{7} \\
& +0.0096655287955 \eta^{8}-0.0019346791089 \eta^{9} \\
& -0.000758100206 \eta^{10}+0.00021161307 \eta^{11}
\end{aligned}
$$

To examine the accuracy of the obtained approximate solution by using the Bernstein and Chebyshev polynomials with operational matrices to solve this problem, we defined the maximal error remainder $M E R_{n}$ as the form

$$
E R_{n}(\eta)=F^{\prime \prime \prime}(\eta)+2 \alpha \operatorname{Re} F(\eta) F^{\prime}(\eta)+(4-H a) \alpha^{2} F^{\prime}(\eta),
$$

and the $M E R_{n}$ is

$$
M E R_{n}=\max _{0 \leq \eta \leq 1}\left|E R_{n}(\eta)\right| .
$$

Figure 5 illustrates that the logarithmic plots for $M E R_{n}$ of the approximate solution gotten by solving this problem by using the operational matrix methods based on the Bernstein or Chebyshev polynomials which indicate the efficiency of these methods. The values of $n$ has been considered $n=4$ to 11 , the efficiency has been seen by the errors will be decreasing when $n$ increasing.

In additional, numerical comparison between the solutions calculated by the suggested methods and the RK4 method when $n=12$. This comparison is presented in Figure 6, the good agreements between the approximate solutions and RK4 can be clearly seen. 


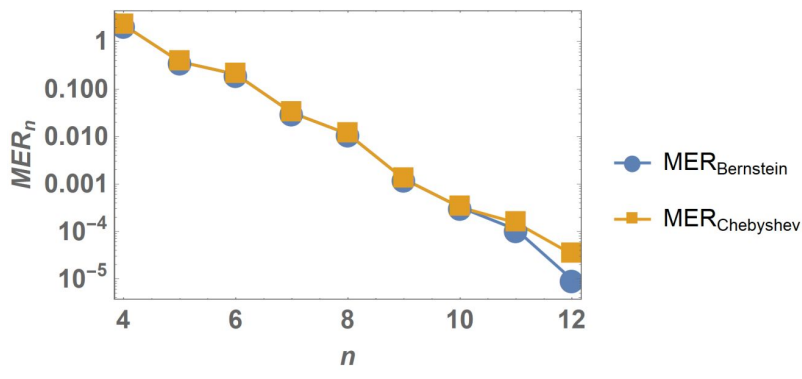

Figure 5: Logarithmic plots for the $M E R_{n}$ for versus n for JefferyHamel fluid flow

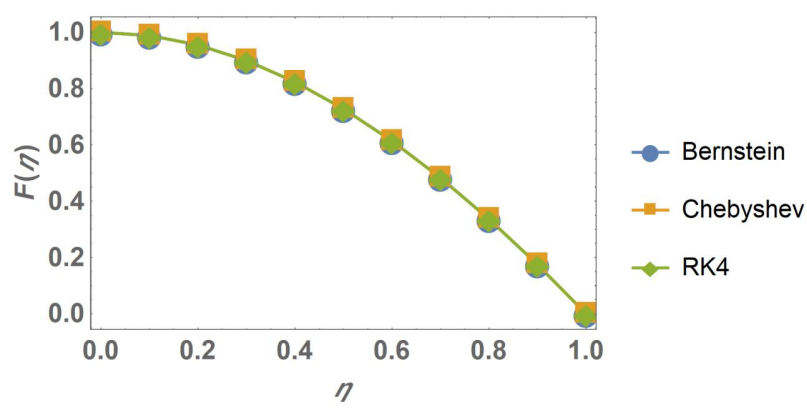

Figure 6: The comparison of the solutions for Jeffery-Hamel fluid flow when $n=12, \alpha=5^{\circ}, \operatorname{Re}=10, H a=0$

\subsection{Straight fin problem}

Nonlinear fin problems are important in engineering and applied sciences because of their applications and the wide uses in the different field of science and technology [17].

The 1D straight fin is presented in this form

$$
S \frac{d}{d x}\left(k(T) \frac{d T}{d x}\right)-p h\left(T-T_{a}\right)=0,
$$

where $k(T)$ is the temperature with heat transfer coefficient or the thermal conductivity, $T$ is the distribution of the heat on the fin, $S$ is some arbitrary constant area (crosssectional), $L$ is the fin length, $p$ is the perimeter, $h$ represents the heat transfer coefficient, $T_{a}$ and $T_{b}$ are temperatures of base surface and surrounding fluid, in a respective way, see Figure 7 [17].

The thermal conductivity of the fin material is assumed to be a linear function of temperature as

$$
k(T)=k_{a}\left(1+\gamma\left(T-T_{a}\right)\right),
$$

where $k_{a}$ is the thermal fin conductivity at $T_{a}$ and $\gamma$ is a parameter without dimensions which represented the variance in the temperature conductivity [18].

$$
u=\frac{T-T_{a}}{T_{b}-T_{a}}, \quad x=\frac{y}{b}, \quad \beta=\gamma\left(T_{b}-T_{a}\right), \mu^{2}=\frac{h p L^{2}}{k_{a} S} u .
$$

The nonlinear equation of the straight energy described as a balance of fins extending to the surface under the influence was obtained by temperature-dependent thermal conductivity.

$$
u^{\prime \prime}(x)+\beta u(x) u^{\prime \prime}(x)+\beta\left(u^{\prime}(x)\right)^{2}-\mu^{2} u(x)=0,
$$

with boundary conditions:

$$
u^{\prime}(0)=0, u(1)=1 \text {, and } u(0)=a,
$$

where $u$ is an unknown function which represents the temperature without dimensions, $\mu$ the parameter of the thermal fin, $\beta$ parameter of the thermal conductivity, $a$ is a parameter can be evaluated, see [19].

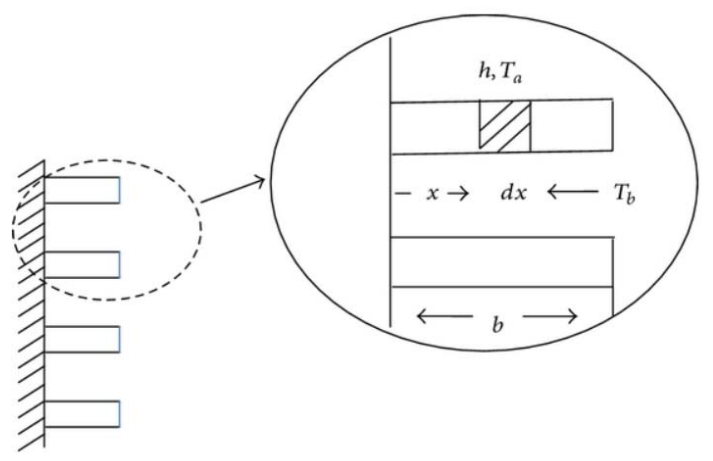

Figure 7: Geometry of the straight fin [17]

\subsubsection{Solving straight fin problem by Bernstein and Chebyshev operational matrices methods}

The operational matrices based on Bernstein and Chebyshev polynomials can be applied to solve the Eq. (44) with boundary conditions Eq. (45).

We will replace the unknown function $u(x)$ and their differentials as an operational matrices form, Eqs. (2) and (8) will be used for the Bernstein polynomial, we get

$$
\begin{gathered}
C^{T}(D)^{2} \Phi(x)+\beta C^{T} \Phi(x) C^{T}(D)^{2} \Phi(x) \\
+\beta\left(C^{T} D \Phi(x)\right)^{2}-\mu^{2} C^{T} \Phi(x)=0, \\
C^{T} \Phi(1)=1, \quad C^{T} D \Phi(0)=1, \quad C^{T} \Phi(0)=a .
\end{gathered}
$$

Eqs. (12) and (17) for the Chebyshev polynomial can be used in Eqs. (44) and (45), we obtain

$C^{T}\left(D^{\star}\right)^{2} \Phi(x)+\beta C^{T} \Phi(x) C^{T}\left(D^{\star}\right)^{2} \Phi(x)+\beta\left(C^{T} D^{\star} \Phi(x)\right)^{2}$ 


$$
-\mu^{2} C^{T} \Phi(x)=0
$$

$$
C^{T} \Phi(1)=1, \quad C^{T} D^{\star} \Phi(0)=1, \quad C^{T} \Phi(0)=a .
$$

The algebraic system obtained by substitute the collocation nodes on the Eqs. (46) and (47).

The following approximate polynomial for this problem when $n=11, \beta=-0.1, \mu=0.4, a=0.917606$, the value of $a$ is taking from [19].

- By using Bernstein polynomial operational matrices

$$
\begin{aligned}
& u(x) \approx 0.917606+1.7763568394 \times 10^{-15} x \\
& +0.0808250335659 x^{2}+2.62673438555 \times 10^{-10} x^{3} \\
& +0.001546176 x^{4}+1.7047113942 \times 10^{-8} x^{5} \\
& +0.000022781048 x^{6}+1.1997076398 \times 10^{-7} x^{7} \\
& +2.541537469 \times 10^{-7} x^{8}+1.16053797 \times 10^{-7} x^{9} \\
& -4.0269924284 \times 10^{-8} x^{10}+8.872746093 \times 10^{-9} x^{11}
\end{aligned}
$$

- By using shifted Chebyshev polynomial operational matrices

$$
\begin{aligned}
& u(x) \approx 0.9176059999846772 \\
& +1.950476985273456 \times 10^{-9} x \\
& +0.08082499289875175 x^{2} \\
& +3.225241157699642 \times 10^{-7} x^{3} \\
& +0.0015449278011654942 x^{4} \\
& +0.000002626330338109295 x^{5} \\
& +0.000019813454762065937 x^{6} \\
& +0.000001782631253763988 x^{7}
\end{aligned}
$$

To examine the accuracy of the obtained approximate solution by using the Bernstein and Chebyshev polynomials with operational matrices to solve this problem, we defined the maximal error remainder $M E R_{n}$ as the form

$$
E R_{n}(x)=u^{\prime \prime}(x)+\beta u(x) u^{\prime \prime}(x)+\beta\left(u^{\prime}(x)\right)^{2}-\mu^{2} u(x),
$$

and the $M E R_{n}$ is

$$
M E R_{n}=\max _{0 \leq x \leq 1}\left|E R_{n}(x)\right|
$$

The logarithmic plots for $M E R_{n}$ of the approximate solution has been shown in Figure 8 by solved this problem using the operational matrix methods for both Bernstein and Chebyshev polynomials which indicate the efficiency of these methods. The values of $n$ has been presented in Figure 8, we taken $n=4$ till 10 , the efficiency has been seen by the errors will be decreasing when $n$ increasing. Also, it can be seen clearly the error of using the Bernstein polynomial is less than using the Chebyshev polynomial this indicates the operational matrix method with Bernstein polynomial provides better accuracy.

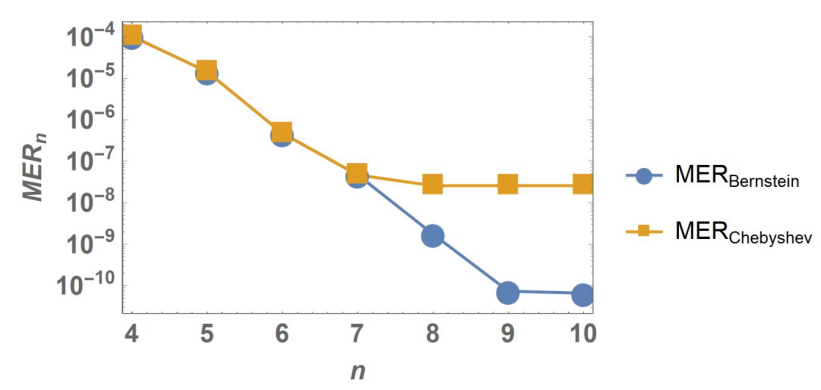

Figure 8: Logarithmic plots for the $M E R_{n}$ for versus $\mathrm{n}$ for straight fin problem

Also, the numerical comparison between the solutions calculated by the suggested methods and the RK4 method when $n=11$ is given in Figure 9. The good agreements between the approximate solutions and RK4 can be noticed.

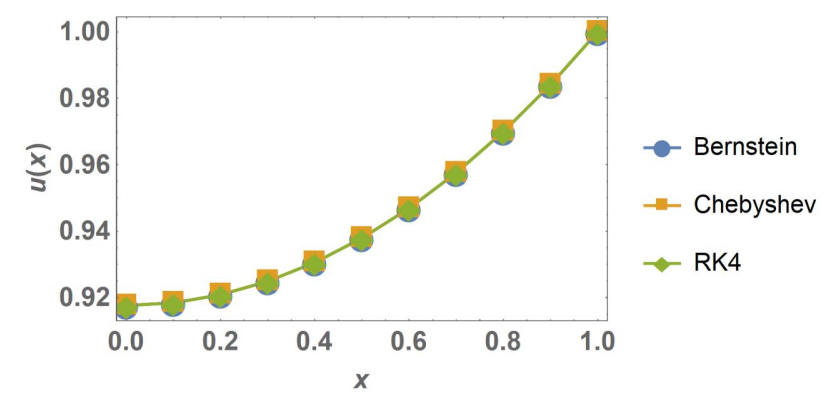

Figure 9: The comparison of the solutions for Straight Fin Problem when $\mathrm{n}=11, \beta=-0.1, \mu=0.4, a=0.917606$.

\subsection{The Falkner-Skan equation}

The Falkner-Skan equation is classified as one of the nonlinear ordinary differential equations of the third order which has a large number of applications, such as insulation materials, glass applications and polymer studies [20].

The Falkner-Skan equation represents in the study of laminar boundary layers displaying similarity. The problem is given by

$$
y^{\prime \prime \prime}(x)+y(x) y^{\prime \prime}(x)+\beta\left[\epsilon^{2}-\left(y^{\prime}(x)\right)^{2}\right]=0,
$$


with boundary conditions as the following:

$$
y(0)=0, \quad y^{\prime}(0)=1-\epsilon, \quad y^{\prime}(\infty)=\epsilon .
$$

where $\beta$ is the pressure gradient parameter and $\epsilon$ is velocity ratio parameter.

When $\beta=0$, Eq (50) is called the Blasius, when $\beta=$ 1, Eq. (50) is called the Hiemenz flow problem and when $\beta=\frac{1}{2}$, Eq. (50) represent to the Homann flow problem.

To solve this problem we can find the numerical value of the missing boundary conditions by using the Padé approximate method. For more details see [21].

Thus, the boundary conditions become

$$
y(0)=0, \quad y^{\prime}(0)=1-\epsilon, \quad y^{\prime \prime}(0)=a .
$$

We will use the value of $a=-0.832666$ as given in [21].

\subsubsection{Solving Falkner-Skan equation by Bernstein and Chebyshev operational matrices methods}

The operational matrices based on Bernstein and Chebyshev polynomials will be used to solve the Eq. (50) with boundary conditions represented in Eq. (52).

The unknown function $y(x)$ and their differentials have been exchanged as an operational matrices form, Eqs. (2) and (8) for the Bernstein polynomial has been used to get equations as the form follows

$$
\begin{aligned}
& C^{T}(D)^{3} \Phi(x)+C^{T} \Phi(x) C^{T}(D)^{2} \Phi(x) \\
& +\beta\left[\epsilon^{2}-\left(C^{T} D \Phi(x)\right)^{2}\right]=0,
\end{aligned}
$$

$$
C^{T} \Phi(0)=0, \quad C^{T} D \Phi(0)=1-\epsilon, \quad C^{T}(D)^{2} \Phi(0)=a
$$

Eqs. (12) and (17) for the Chebyshev polynomial can be used in Eqs.(50) and (52), we obtain

$$
\begin{gathered}
C^{T}\left(D^{\star}\right)^{3} \Phi(x)+C^{T} \Phi(x) C^{T}\left(D^{\star}\right)^{2} \Phi(x) \\
+\beta\left[\epsilon^{2}-\left(C^{T} D^{\star} \Phi(x)\right)^{2}\right]=0, \\
C^{T} \Phi(0)=0, \quad C^{T} D^{\star} \Phi(0)=1-\epsilon, \quad C^{T}\left(D^{\star}\right)^{2} \Phi(0)=a .
\end{gathered}
$$

The algebraic system has been achieved by substituting the collocation nodes on the Eqs. (53) and (54).

We consider $n=4$ to 12 in this problem. If $n=12, \beta=$ $0.5, \epsilon=0.1, a=-0.832666$, (for calculate the value of a, see [21]) the following approximate polynomial has been gotten
- By using Bernstein polynomial operational matrices

$$
\begin{aligned}
& y(x) \approx 0.89999999999 x \\
& -0.41633299999999807 x^{2} \\
& +0.06666667128520487 x^{3} \\
& -4.104200002075231 \times 10^{-8} x^{4} \\
& -0.0029997785484283668 x^{5} \\
& +0.00046182400910765864 x^{6} \\
& +0.0001628731071718903 x^{7} \\
& -0.00007702244352003618 x^{8} \\
& +0.000004539459496299969 x^{9} \\
& +0.000005072552994533908 x^{10} \\
& -0.000001637857849345891 x^{11} \\
& +1.70446526226442 \times 10^{-7} x^{12}
\end{aligned}
$$

- By using shifted Chebyshev polynomial operational matrices

$$
\begin{aligned}
& y(x) \approx 9.04174260000537 \times 10^{-11} \\
& +0.8999999853077153 x \\
& -0.41633260693889024 x^{2} \\
& +0.06666261954320185 x^{3} \\
& +0.00002086400995171111 x^{4} \\
& -0.0030602804620039276 x^{5} \\
& +0.0005641848447672726 x^{6} \\
& +0.0000635987207972879 x^{7} \\
& -0.00002869443212259562 x^{8}
\end{aligned}
$$

To check the accuracy of the gotten approximate solution by using the Bernstein or Chebyshev polynomials with operational matrices to solve this problem, the maximal error remainder $M E R_{n}$ has been defined as the form

$$
E R_{n}(x)=y^{\prime \prime \prime}(x)+y(x) y^{\prime \prime}(x)+\beta\left[\epsilon^{2}-\left(y^{\prime}(x)\right)^{2}\right],
$$

and the $M E R_{n}$ is

$$
M E R_{n}=\max _{0 \leq x \leq 1}\left|E R_{n}(x)\right|
$$

The logarithmic plots for $M E R_{n}$ of the approximate solution by solved this problem by using the operational matrix methods based on the Bernstein or Chebyshev polynomials have been given in Figure 10 which indicates the efficiency of these methods. We can see the efficiency for these methods when $n$ is increasing, the errors reminders are decreasing. Moreover, the operational matrix method 


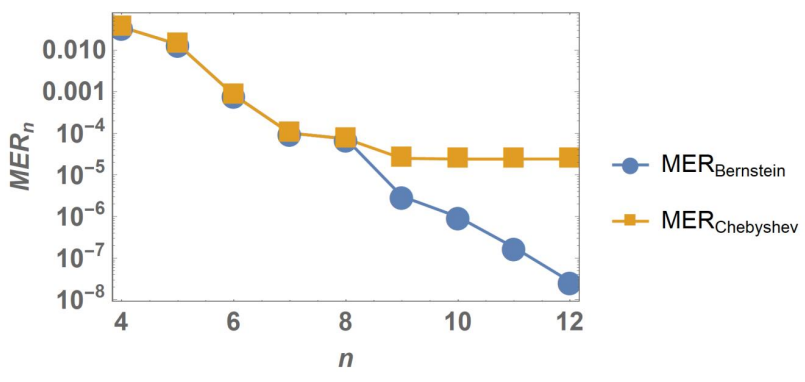

Figure 10: Logarithmic plots for the $M E R_{n}$ for versus $\mathrm{n}$ for FalknerSkan problem

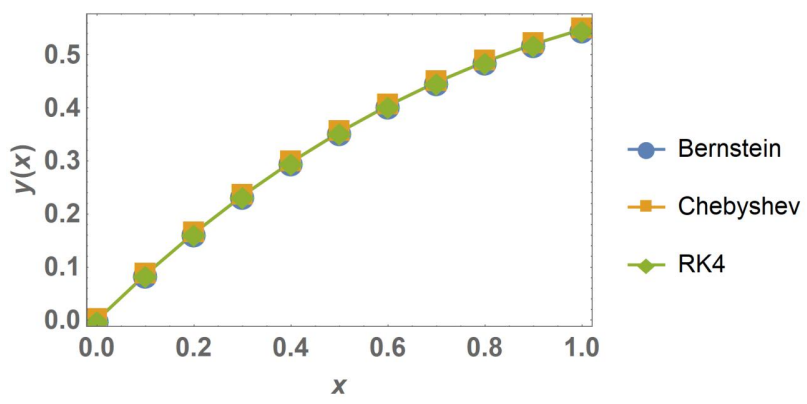

Figure 11: The comparison of the solutions Falkner-Skan problem when $n=12, \beta=0.5, \in=0.1, a=-0.832666$.

based on the Bernstein polynomial provides better accuracy.

In addition, the comparison numerically between the solutions calculated by the suggested methods and the RK4 method when $n=12$, has been presented in Figure 11 . Furthermore, the good agreements between the approximate solutions and RK4 can be seen clearly.

Also, we have calculated the run time of the proposed methods and RK4. It can be seen from Table 1 that running time for the proposed methods are faster compared to the RK4 method in seconds.

Table 1: The comparison of run time between the proposed methods and RK4 per seconds

\begin{tabular}{lccc}
\hline & Time of RK4 & $\begin{array}{c}\text { Time of } \\
\text { Bernstein for } \\
\mathbf{N = 5}\end{array}$ & $\begin{array}{c}\text { Time of Chebyshev } \\
\text { for N=5 }\end{array}$ \\
\hline $\begin{array}{l}\text { MHD squeezing } \\
\text { fluidproblem }\end{array}$ & 0.36 & 0.297 & 0.265 \\
Jeffery -Hamel fluid flow & 0.36 & 0.344 & 0.343 \\
Straight Fin Problem & 0.343 & 0.312 & 0.313 \\
Falkner-Skan problem & 0.328 & 0.297 & 0.265 \\
\hline
\end{tabular}

\section{Conclusion}

In this paper, the methods of operational matrices based on the two different types of Bernstein and Chebyshev polynomials to solve several problems containing NODEs have been used. Mentioned problems arise in engineering and applied sciences. Each problem has been solved and an approximate solution has been obtained by approximate the unknown function by the polynomial series and uses the operational matrices to eliminate of the differentiation from the equation. Moreover, the problems have been solved numerically by using the RK4 method to compare the numerical result with the approximate solutions and the compatibility was good among them. Furthermore, both methods have provided a good accuracy, however, the operational matrix method based on the Bernstein polynomial provides better accuracy. Mathematica ${ }^{\circledR}$ 10 software has been used for calculations on this study.

\section{References}

[1] Jordan D.W., Smith P., Nonlinear Ordinary Differential Equations An introduction for Scientists and Engineers, 4th ed., 2007, Oxford University Press Inc., New York.

[2] Yousefi S.A., Behroozifar M., Operational Matrices of Bernstein Polynomials and Their Applications, Int. J. Sys. Sci., 2010, 41, 709-716.

[3] Ali M.N.M., A New Operational Matrix of Derivative for Orthonormal Bernstein Polynomial's, Baghdad Sci. J., 2014, 1, 1295-1300.

[4] Alshbool M.H.T., Bataineh A.S., Hashim I., Isik O.R., Approximate Solutions of Singular Differential Equations with Estimation Error by Using Bernstein Polynomials, Int. J. Pure Appl. Math., 2015, 100, 109-125.

[5] Pandy P., Chandrakumar R.D., A Computational Method for Solving a Class of Singular Boundary Value Problems Arising in Science and Engineering, Egypt. J. Basic Appl. Sci., 2016, 3, 383-391.

[6] Khataybeh S.N., Hashim I., Alshbool M., Solving Directly ThirdOrder ODEs Using Operational Matrices of Bernstein Polynomials Method with Applications to Fluid Flow Equations, J. King Saud Univ. - Sci., 2019, 31, 4, 822-826.

[7] Sharma B., Kumar S., Paswan M.K., Mahato D., Chebyshev Operational Matrix Method for Lane-Emden Problem, Nonlin. Eng., 2019, 8, 1-9.

[8] Öztür Y., Numerical Solution of Systems of Differential Equations Using Operational Matrix Method with Chebyshev Polynomials, J. Taibah Univ. Sci., 2018, 12, 155-162.

[9] Rajeev N.K.R., A Numerical Solution Based On Operational Matrix of Differentiation of Shifted Second Kind Chebyshev Wavelets for a Stefan Problem, Int. J. Math. Comput. Sci., 2015, 9, 374-377.

[10] Hashemizadeh E., Mahmoudi F., A Numerical Method For Solving Physiology Problems By Shifted Chebyshev Operational 
Matrix, Int. J. Industr. Math., 2018, 10, 95-102.

[11] Al-A'asam J.A., Deriving the Composite Simpson Rule by Using Bernstein Polynomials for Solving Volterra Integral Equations, Baghdad Sci. J,, 2014,11, 1274-1282.

[12] Atabakzadeh M.H., Akrami M.H., Erjaee G.H., Chebyshev Operational Matrix Method for Solving Multi-Order Fractional Ordinary Differential Equations, Appl. Math Model., 2013, 37, 8903-8911.

[13] Ullah I., Rahim M.T., Khan H., Qayyum M., Analysis of Various Semi-Numerical Schemes for Magnetohydrodynamic (MHD) Squeezing Fluid Flow in Porous Medium, Propul. Power Res., 2019, 8, 69-78.

[14] Ullah I., Rahim M.T., Khan H., Qayyum M., Analytical Analysis of Squeezing Flow in Porous Medium with MHD Effect, UPB Sci. Bulletin, Series A: Appl. Math. Phys., 2016, 78, 2.

[15] AL-Jawary M.A., Abdul Nabi A.J., Three Iterative Methods for Solving Jeffery-Hamel Flow Problem, Kuwait J. Sci., 2020, 47, 1-13.
[16] Ganji D.D., Hatami M., Three Weighted Residual Methods Based on Jeffery-Hamel Flow, Int. J. Numer. Meth. Heat Fluid Flow, 2014, 24, 654-668.

[17] Sevilgen G., A Numerical Analysis of a Convective Straight Fin With Temperature-Dependent Thermal Conductivity, Thermal Sci., 2017, 21, 939-952.

[18] Akindeinde S.O., Parker-Sochacki Method for the Solution of Convective Straight Fins Problem With TemperatureDependent Thermal Conductivity, Int. J. Nonlin. Sci., 2018, 20, 1-11.

[19] Abdul Nabi A.J., AL-Jawary M.A., Reliable Iterative Methods for Solving Convective Straight and Radial Fins with TemperatureDependent Thermal Conductivity Problems, Gazi Univ. J. Sci., 2019, 32, 967-989.

[20] Bakodah H.O., Ebaid A., Wazwaz A.M., Analytical and Numerical Treatment of Falkner-Skan Equation Via a Transformation and Adomian's Method, Roman. Rep. Phys., 2018, 70, 111, 1-17.

[21] Al-Jawary M.A., Adwan M.I., Reliable iterative methods for solving the Falkner-Skan equation, Gazi Univ. J. Sci, 2020, 33, $168-186$. 